\title{
A case of viable fetus co-existing with a complete hydatidiform mole in a twin pregnancy with successful outcome
}

\author{
Seneesh Kumar Vikraman*, Vipin Chandra, Bijoy Balakrishanan, \\ Meenu Batra, Rekha Kuriakose, Gopinathan Kannoli
}

\begin{abstract}
Department of Feto-Maternal Medicine, Obstetrics and Gynecology, CIMAR Edappal, Edappal Hospitals Pvt Ltd, Edappal, Malappuram, Kerala, India
\end{abstract}

Received: 25 November 2014

Accepted: 20 December 2014

\section{*Correspondence:}

Dr. Seneesh Kumar Vikraman,

E-mail: drseneeshkv@gmail.com

Copyright: (C) the author(s), publisher and licensee Medip Academy. This is an open-access article distributed under the terms of the Creative Commons Attribution Non-Commercial License, which permits unrestricted non-commercial use, distribution, and reproduction in any medium, provided the original work is properly cited.

\begin{abstract}
Twin pregnancies with a viable fetus and co-existing complete hydatidiform mole (CHMF) are being increasingly reported in literature. It is now evident that with proper obstetric care live births can be reasonably expected. We present here such a case successfully managed at our centre with one year follow up of mother and the baby. The case highlights the role of prompt diagnosis, rational prenatal counselling, evidence based management and role of high risk obstetric and neonatal care in providing solutions to seemingly difficult situations.
\end{abstract}

Keywords: Hydatidiform mole, Twin pregnancy, Complete mole, Placental echoes, Karyotype

\section{INTRODUCTION}

Gestational trophoblastic disease includes hydatidiform mole (complete and partial) and gestational trophoblastic neoplasia (invasive mole, choriocarcinoma, placental site trophoblastic tumour, and epithelioid trophoblastic tumour). ${ }^{1}$

Twin pregnancy with a complete hydatidiform mole and a normal fetus (CHMF) occurs with an estimated incidence of one in 22000-100000 pregnancies. ${ }^{2}$ Partial molar pregnancy with coexisting fetus is a rare complication of pregnancy with the incidence of $0.005 \%$ to $0.01 \%{ }^{3}$ Hydatidiform mole refers to an abnormal pregnancy characterized by varying degrees of trophoblastic proliferation (both cytotrophoblast and syncytiotrophoblast) and vesicular swelling of placental villi associated with an absent or an abnormal fetus/embryo. Complete mole refers to when there is total replacement of normal placenta by grossly dilated and hydropic villi in the absence of fetus and incomplete or partial mole when there is partial replacement with hydropic villi and visible abnormal fetal parts. ${ }^{4}$

Cases of association of CHMF can be classified into three types. The first is a twin pregnancy with one normal fetus (normal placenta) and another complete mole, the second is a twin pregnancy with one normal fetus (normal placenta) and another partial mole and the third is a singleton normal fetus with partial molar placenta. ${ }^{5}$

Approximately $90 \%$ of complete moles are $46 \mathrm{XX}$, originating from duplication of the chromosomes of a haploid sperm after fertilization of an egg in which the maternal chromosomes are either inactive or absent. The other $10 \%$ of complete moles are $46 \mathrm{XY}$, or $46 \mathrm{XX}$, as a result of fertilization of an empty ovum by 2 sperm (dispermy). ${ }^{6}$

Partial hydatidiform moles demonstrate identifiable fetal or embryonic tissue, chorionic villi with focal edema that vary in size and shape, scalloping and prominent stromal trophoblastic inclusions, and a functioning villous 
circulation, as well as focal trophoblastic hyperplasia with mild atypia only. Most partial moles have a triploid karyotype (usually 69, XXY), resulting from the fertilization of an apparently normal ovum by 2 sperm.

Case reports of CHMF are increasingly being reported in the literature with successful feto-maternal outcome in many of them.

\section{CASE REPORT}

A 26 year old Indian woman, gravida 2 para 1 with a previous intra-uterine demise at 29 weeks of gestation of undetermined cause, presented at our clinic at 14 weeks and 3 days gestation with an ultrasound report suspicious of a pregnancy with co-existing complete hydatidiform mole (CHM). An ultrasound performed revealed a viable pregnancy corresponding to the gestational age with a separate well-defined multiple cystic, snowstorm-like mass connected with the normal placenta (Figure 1). A serum quantitative hCG was $280000 \mathrm{IU} / \mathrm{L}$. A diagnosis of CHMF was made based on these findings.

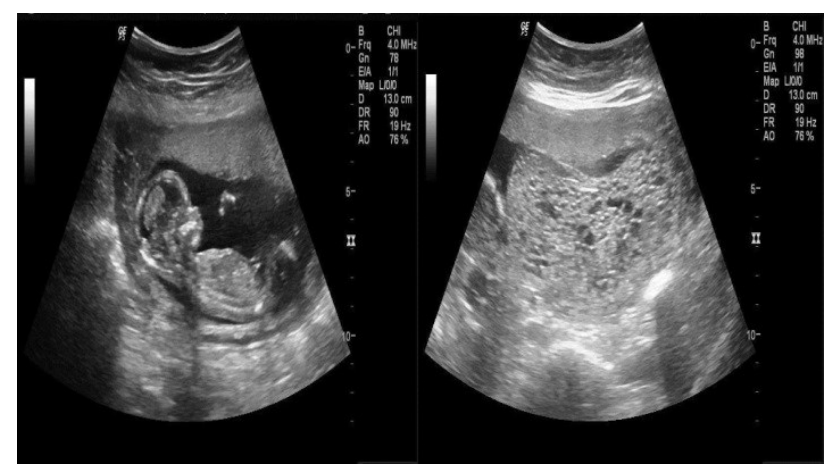

Figure 1: Ultrasound image showing snowstorm pattern of complete hydatidiform mole with a viable co-existing fetus at 14 weeks 3 days.

The couple were informed about the scan findings and non- directive counselling was performed about the situation and the risks involved. The couple opted for continuation of pregnancy with a decision to stay near the premises of the hospital. Baseline investigations revealed a maternal blood group of B plus with mild anemia and hyperthyroidism. Hematinics and Propylthiouracil (PTU) were started.

Case was reviewed at 16 weeks .Amniocentesis was done from the sac with viable fetus for fetal karyotype. The latter was found to be normal.

She was managed in the high risk pregnancy unit of our hospital. Medications included haematinics, progestogens and PTU. Target scan performed at 20 weeks was within normal limits. Serial surveillance was done. Scan included fetal wellbeing, cervical length assessment and inspection of CHM. Antenatal steroids were administered at 24 weeks.
At 28 weeks and 2 days she developed severe intractable ante partum haemorrhage. Emergency caesarean section was performed with the birth of live female baby weighing 806 grams with APGAR of 6/10 at 5 min and $7 / 10$ at $10 \mathrm{~min}$ and two placenta, one apparently normal and other showing features of CHM. Baby was shifted to NICU. CHM placenta was sent for histopathology and karyotype. CHM was confirmed and karyotype was 46 XX.

Finally both mother and baby were discharged on from the hospital in sound health. Mother's serum beta HCG was serially monitored till one year and was undetectable. Baby had normal attainment of milestones and growth till last follow up.

\section{DISCUSSION}

Abnormal placental echoes, raised beta hCG levels detected during aneuploidy screening, persistent hyperemesis gravidarum and unexplained vaginal bleeding usually raises suspicion of CHMF. All cases of twin pregnancies (or higher order) with co existent gestational trophoblastic disease need a careful introspection to distinguish between $\mathrm{CHM}$ and partial mole. In our case the ultrasound features were classical and adequate counselling could be done which ensured full co-operation from the couple.

Twin pregnancy with complete hydatidiform mole has a higher risk of maternal complications than partial hydatidiform moles. When a fetus is present in conjunction with partial mole, it generally exhibits the stigmata of triploidy, including growth restriction and multiple congenital malformations. ${ }^{7}$ However term pregnancies with partial moles with normal fetal outcomes have been reported. ${ }^{8}$

Maternal complications in CHMF include Preeclampsia, hyperthyroidism, anemia, hyperemesis gravidarum, antepartum haemorrhage and persistent trophoblastic disease. Fetal complications include early pregnancy losses, late fetal demise, chromosomal aberrations, congenital anomalies, fetal growth restriction, prematurity and neonatal thyrotoxicosis 9,10 with spontaneous fetal loss being the most likely outcome. ${ }^{11}$

It should be noted that the couple who already had a previous pregnancy loss, were ready to stay near the premises of the hospital. This considerably reduced their anxiety as they were assured of round the clock help in case need arises. Fetal karyotype and target scan ensured euploid and anatomically normal fetus which further boosted the morale of the couple.

An elevated beta-hCG with low serum placental lactogen values, snow-storm appearance of placenta in the USG helps to diagnose the above condition. Histopathology and cytogenetics helps in the final diagnosis. However the sonographic appearance may be less marked in partial mole. 
A partial mole is usually distinguishable from a CHM by DNA polymorphism analysis. Chorionic villus sampling of molar tissue can be performed and absence of maternal alleles confirms a diandrogenic complete mole. ${ }^{11}$ Thyroid function test should be done. Fetal karyotype is mandatory. Early target scan needs to be performed. Early prophylactic antenatal steroids in view of increased risk of pre-term labour is a recommended. After delivery serial follow up of mother for early detection of persistent trophoblastic disease should be done.

Institutional care with facilities of good neonatal unit is essential. With systematic supervision and high risk surveillance good live birth rates can be expected.

Funding: No funding sources

Conflict of interest: None declared

Ethical approval: Not required

\section{REFERENCES}

1. John R. Lurain. Gestational trophoblastic disease I: epidemiology, pathology, clinical presentation and diagnosis of gestational trophoblastic disease, and management of hydatidiform mole. Am J Obstet Gynecol. 2010;203(6):531-9.

2. Antonella Vimercati. Two cases of complete hydatidiform mole and coexistent live fetus. J Prenat Med. 2013;7(1):1-4.

3. Suzuki M, Matsunobu A, Vakita K, Nishijima M, Osanai K. Hydatidiform mole with surviving coexistent fetus. Ombt Gynecol. 1980;56:384-8.

4. Alessandro Cavaliere, Santina Ermito, Angela Dinatale, Rosa Pedata. Management of molar pregnancy. J Prenat Med. 2009;3(1):15-7.

5. Hsieh CC, Hsieh TT, Hsueh C, Kuo DM, Lo LM, Hung TH. Delivery of a severely anemic fetus after partial molar pregnancy: clinical and ultrasonographic findings. Hum Reprod. 1999;14(4):1122-6.

6. John R. Lurain. Gestational trophoblastic disease I: epidemiology, pathology, clinical presentation and diagnosis of gestational trophoblastic disease, and management of hydatidiform mole. Am J Obstet Gynecol. 2010;203(6):531-9.

7. Lawler SD, Fisher RA, Dent J. A prospective genetic study of complete and partial hydatidiform moles. Am J Obstet Gynecol. 1991;164:1270-7.

8. Shobha UN, Dhananjaya BS, Sunil Kumar Nanda, Nanda Gopal, Tejeswini KK, Musarrat YA. Term pregnancy with partial molar changes: a case report. Int J Biol Med Res. 2011;2(4):1191-2.

9. Unsal MA, Guven S. Complete hydatidiform mole coexisting with a live fetus. Clin Exp Obstet Gynecol. 2012;39(2):262-4.

10. Kutuk MS, Ozgun MT, Dolanbay M, Batukan C, Uludag S, Basbug M. Sonographic findings and perinatal outcome of multiple pregnancies associating a complete hydatiform mole and a live fetus: a case series. J Clin Ultrasound. 2014;42(8):465-71.

11. Wax JR, Pinette MG, Chard R, Blackstone J, Cartin A. Prenatal diagnosis by DNA polymorphism analysis of complete mole with coexisting twin. Am J Obstet Gynecol. 2003;188:1105-6.

DOI: $10.5455 / 2320-1770 . i j r \operatorname{cog} 20150252$

Cite this article as: Vikraman SK, Chandra V, Balakrishanan B, Batra M, Kuriakose R, Kannoli G. A case of viable fetus co-existing with a complete hydatidiform mole in a twin pregnancy with successful outcome. Int J Reprod Contracept Obstet Gynecol 2015;4:266-8. 